\title{
Peculiaridades do transtorno obsessivo-compulsivo na infância e na adolescência
}

\author{
M aria Conceição do Rosario-Campos
}

Study Center da Universidade de Yale. New Haven, CT, EUA. Protoc (Projeto Transtornos do Espectro Obsessivo-Compulsivo) do Hospital das Clínicas da Faculdade de Medicina da USP (HC-FM/USP). São Paulo, SP, Brasil. Departamento de Psiquiatria da FM/USP. São Paulo, SP, Brasil

\begin{abstract}
Obsessive-compulsive disorder (OCD) has a bimodal age of onset and a range of treatment outcomes. Although most of the studies carried out so far have considered childhood and adult forms of OCD as the same disorder, more recent data have suggested that $O C D$ children, as well as adults with an early onset of their obsessive-compulsive symptoms, may represent a distinct subgroup. This review briefly summarizes the most common clinical characteristics of the OCD presentation in children and adolescents, shows data reinforcing the idea that age of onset may be an important distinguishing feature and discusses the importance of a systematic assessment of age of onset for identifying more homogeneous subgroups of OCD patients.
\end{abstract}

Keywords Obsessive-compulsive disorder. Age of onset. Child psychiatry. Adolescence.

\section{Introdução}

Um longo caminho tem sido percorrido desde a primeira descrição da síndrome obsessivo-compulsiva na infância por Pierre Janet, em 1903. Passos importantes foram o reconhecimento de que, apesar de considerado inicialmente um transtorno raro em crianças, as taxas de prevalência do transtorno obsessivo-compulsivo (TOC) na infância e adolescência são semelhantes às taxas na idade adulta, variando de $1,9 \mathrm{a} 4,0 \%,{ }^{1} \mathrm{e}$ aproximadamente $1 / 3$ dos pacientes adultos apresenta o início dos sintomas na infância. ${ }^{2}$

Apesar do aumento considerável no número de estudos envolvendo crianças e adolescentes com TOC, questões importantes ainda continuam sem respostas. Por exemplo: qual a melhor maneira de determinar a idade de início do quadro? A idade de início dos sintomas obsessivo-compulsivos (SOC) é importante para a determinação de subgrupos de pacientes? As peculiaridades da apresentação do TOC em crianças estariam restritas à infância ou persistiriam até a idade adulta? Qual a importância dessas peculiaridades para a investigação de fatores etiopatogênicos e preditivos de resposta ao tratamento? Qual a idade adequada para se considerar início precoce do TOC? Pacientes adultos, mas com início precoce do quadro, pertencem ao mesmo subgrupo de crianças com TOC?

Este artigo apresenta algumas características da apresentação do TOC na infância, além de resultados de estudos mais recentes com adultos em que os dados foram analisados de acordo com a idade de início dos SOC.

\section{TOC na infância e TOC de início precoce}

Antes da puberdade, há um predomínio de meninos diagnosticados com TOC. Na adolescência, há um aumento do número de casos entre meninas, chegando a uma proporção de $1: 1$ na idade adulta. ${ }^{1}$

O DSM-IV e a CID-10 aplicam os mesmos critérios diagnósticos para adultos e crianças, com exceção da necessidade de reconhecimento de que os SOC são excessivos ou irracionais. A ausência desse critério reflete achados da literatura de que cerca de $30 \%^{3}$ a $50 \%{ }^{4}$ das crianças têm a crítica, em relação aos sintomas, parcial ou totalmente comprometida.

Não existe um padrão único de história natural dos SOC. O início do quadro pode ser abrupto ou insidioso e pode ou não estar relacionado a algum fator precipitante, além de os sintomas tenderem a se modificar ao longo do tempo. Crianças são geralmente ainda mais sigilosas em relação aos sintomas do que os adultos. A maioria dos estudos relata um intervalo médio entre o início dos SOC e a data da primeira avaliação variando de 2,5 anos em crianças $^{5}$ a 16,3 anos em adultos com início precoce. $^{6}$

Entre os sintomas freqüentes, encontram-se medo de contaminação, de ferir-se ou ferir outras pessoas, obsessões sexuais e de simetria, compulsões de lavagem, verificação, repetição, contagem, ordenação/arranjo e compulsões semelhantes a tiques ("tic-like"). Apesar de a maioria das crianças apresentar múltiplas obsessões e compulsões, é comum as compulsões precederem o início das obsessões, além de ser mais comum encontrar compulsões sem obsessões em crianças do que em adolescentes. ${ }^{7}$

Um estudo com pacientes adultos, divididos de acordo com a idade de início dos SOC, encontrou que, no grupo de início precoce, as compulsões começaram em média dois anos antes, ao passo que, no grupo de início tardio, obsessões e compulsões começaram ao mesmo tempo. O grupo de início precoce 
apresentou também maior freqüência de compulsões "tic-like”, de repetição e de colecionamento, além de maior gravidade na subescala de compulsões da Yale-Brown Obsessive-Compulsive Scale, Y-BOCS. ${ }^{6}$

\section{Comorbidade}

Assim como adultos com TOC, crianças e adolescentes apresentam altas taxas de comorbidade. Geller et al $^{3}$ encontraram que $90 \%$ das crianças e adolescentes apresentavam outros transtornos co-mórbidos, sendo que $70 \%$ preenchiam critérios para transtornos disruptivos do comportamento. Além dos quadros depressivos e fobias simples (presentes também em adultos), ansiedade de separação, transtornos disruptivos e tiques são freqüentes na infância. ${ }^{8}$

Apesar das tentativas de determinar subgrupos a partir desses quadros co-mórbidos, apenas a associação entre TOC e tiques tem sido comprovada por estudos clínicos, genéticos e de neuroimagem. ${ }^{9}$ Em crianças, essa associação é ainda mais freqüiente, com taxas variando de $20 \%$ a $59 \% .{ }^{10}$ Rosario-Campos et al* relataram que $47 \%$ dos pacientes adultos, mas com início precoce, apresentaram tiques e/ou síndrome de Tourette (ST), comparados com $9 \%$ dos pacientes com início tardio dos SOC.

\section{Estudos genéticos}

Até o momento, foram publicados 19 estudos genéticofamiliares com pacientes com TOC, apresentando resultados discordantes. Destes, apenas três coletaram os dados a partir de crianças e adolescentes. ${ }^{11-13}$ Em relação à heterogeneidade das amostras, acredita-se que quanto mais precoce é o início dos SOC nos pacientes, maior o risco de morbidade para TOC entre os familiares. De maneira semelhante ao TOC, formas subclínicas também foram transmitidas. ${ }^{14}$

Estudos com pacientes com ST têm fornecido evidências adicionais da transmissão e expressão genética do TOC. Esses estudos revelam não apenas taxas aumentadas de SOC e TOC em familiares de pacientes com $\mathrm{ST}^{15}$ mas também aumento de tiques e/ou ST em parentes de pacientes com TOC. ${ }^{13}$ Quanto mais precoce o início dos SOC nos probandos, maior a probabilidade de os familiares apresentarem tiques e/ou ST. ${ }^{14}$

\section{Tratamento}

Tanto os inibidores da recaptação de serotonina (IRS) quanto as psicoterapias de base cognitiva e/ou comportamental têm tido resultados positivos no tratamento de crianças e adolescentes com TOC.

Deve-se começar com uma avaliação abrangente da criança e de sua família, com os objetivos de determinar sintomasalvo e grau de interferência destes no funcionamento escolar, familiar e social, de estabelecer um bom vínculo com a criança, esclarecer a respeito da origem do quadro e fornecer suporte e orientação familiar.

A terapia cognitivo-comportamental (TCC) melhora os SOC e diminui o risco de recaída após a retirada da medicação, devendo ser considerada como tratamento de escolha na infância. ${ }^{8}$ A introdução imediata de IRS deve ser feita apenas quando os sintomas estão muito graves, quando houver risco de suicídio, na presença de quadros co-mórbidos associados ou quando o nível de ansiedade estiver alto o suficiente para impedir a realização de TCC.

Independentemente do IRS utilizado, é importante iniciar seu uso com doses baixas e aumentos gradativos. Dosagens freqüentemente utilizadas estão descritas na Tabela. Para avaliação de sua eficácia, deve-se manter a medicação por pelo menos três meses em dose máxima. Caso os sintomas estejam controlados, pode-se começar a redução para uma dosagem de manutenção após seis meses e tentar sua suspensão após 18 meses. ${ }^{16}$ Deve-se retirar a medicação lentamente, reduzindo $25 \%$ a cada dois meses.

\begin{tabular}{lcc}
\hline \multicolumn{2}{l}{ Tabela - Doses freqüentemente utilizadas (Grados et al, ${ }^{\mathbf{1 8}} \mathbf{1 9 9 9 )}}$. \\
Clomipramina & Dose inicial & Variação de dosagem \\
Sertralina & $25 \mathrm{mg}$ & $50-200 \mathrm{mg} / \mathrm{dia}$ \\
Fluvoxamina & $12,5-25 \mathrm{mg}$ & $50-200 \mathrm{mg} / \mathrm{dia}$ \\
Fluoxetina & $12,5-25 \mathrm{mg}$ & $50-200 \mathrm{mg} / \mathrm{dia}$ \\
Paroxetina & $5-10 \mathrm{mg}$ & $5-50 \mathrm{mg} / \mathrm{dia}$ \\
Citalopram & $10-20 \mathrm{mg}$ & $10-40 \mathrm{mg} / \mathrm{dia}$ \\
\hline
\end{tabular}

No caso da clomipramina, o monitoramento cardíaco criterioso deve ser realizado, além da importância de evitar interação medicamentosa com antibióticos. ${ }^{17}$

Parâmetros clínicos de resposta ao tratamento incluem maior tempo gasto com os SOC, interferência nas atividades escolares e de vida diária, não-reconhecimento de que os sintomas são excessivos ou irracionais, pior resposta inicial ao tratamento, história de tiques e/ou ST, presença de doença psiquiátrica na família e problemas familiares. ${ }^{7}$

Apenas três estudos clínicos com pacientes adultos avaliaram seus resultados de acordo com a idade de início dos SOC, sendo que pacientes com início precoce tiveram uma pior resposta ao tratamento com clomipramina ${ }^{18,19}$ ou IRS.*

\section{Conclusões}

De forma geral, a apresentação do TOC é semelhante nas diversas faixas etárias. Talvez por essa razão, a maioria das pesquisas considere igualmente o TOC na infância e na idade adulta. No entanto, estudos mais recentes nos quais os dados foram analisados de acordo com a idade de início dos SOC têm apresentado características distintas entre as apresentações do TOC na infância e em pacientes com início do quadro após a adolescência, reforçando a idéia de que o TOC de início precoce corresponda a um possível subgrupo de pacientes.*

Infelizmente, ainda não existe consenso sobre como avaliar a idade de início dos SOC e quando considerar início precoce do TOC. Alguns estudos propõem que a idade de início do quadro deve ser avaliada de acordo com o surgimento dos SOC. Outros autores propõem considerar a idade em que os sintomas começam a interferir com o funcionamento da pessoa. Outros sugerem que deveria ser avaliada a partir do momento em que o paciente procura alguma forma de tratamento. ${ }^{6}$

*Rosario-Campos MC, Leckman JF, Mercadante MT, Shavitt RG, Prado HS, Sada P, et al. Adults with Early-Onset Obsessive-Compulsive Disorder. Am J Psychiatry (in press). 
A identificação de subgrupos mais homogêneos de pacientes é de extrema importância para a descoberta de genes envolvidos na manifestação do TOC, para a compreensão de como esses genes interagem com fatores ambientes e neuroquímicos para a etiopatogenia do quadro e para o estabelecimento de formas de tratamento mais eficazes. Para identificar esses subgrupos, estudos longitudinais, assim como trabalhos avaliando a idade de início dos sintomas de forma sistematizada, serão necessários.

\section{Agradecimento}

A Obsessive-Compulsive Foundation pelo financiamento fornecido.

\section{Referências}

1. Zohar AH. The epidemiology of obsessive-compulsive disorder in children and adolescents. Child Adolesc Psychiatr Clin N Am 1999;8:445-60.

2. Geller D, Biederman J, Jones J, Park K, Schwartz S, Shapiro S, et al. Is juvenile obsessive-compulsive disorder a developmental subtype of the disorder? A review of the pediatric literature. J Am Acad Child Adolesc Psychiatry 1998;37:420-7.

3. Geller DA, Biederman J, Griffin S, Jones J, Lefkowitz TR. Comorbidity of juvenile obsessive-compulsive disorder with disruptive behavior disorders. J Am Acad Child Adolesc Psychiatry 1996;35:1637-46.

4. Allsopp M, Verduyn C. Adolescents with obsessive-compulsive disorder: a case note review of consecutive patients referred to a provincial regional adolescent psychiatric unit. J Adolesc 1990;13:157-69.

5. Geller DA, Biederman J, Jones J, Shapiro S, Schwartz S, Park KS. Obsessive-compulsive disorder in children and adolescents: a review. Harv Rev Psychiatry 1998;5:260-73.

6. Rosario-Campos MC. Transtorno obsessivo-compulsivo de início precoce e início tardio: características clínicas, psicopatológicas e de comorbidade [dissertação]. São Paulo: Faculdade de Medicina da Universidade de São Paulo; 1998.

7. March JS, Leonard HL. Obsessive-Compulsive Disorder in Children and Adolescents: A Review of the Past ten years. J Am Acad Child Adolesc Psichiatry 1996;34:1265-73.

8. Piacentini J. Cognitive Behavioral Therapy of Childhood OCD. Child And Adolesc Psychiatr Clin N Am 1999;8:599-616.

9. Miguel EC, Rosario-Campos MC, Prado HS, Valle R, Rauch SL, Coffey BJ, et al. Sensory phenomena in obsessive-compulsive disorder and Gilles de la Tourette syndrome. J Clin Psychiatry 2000;61:150-6.
10. Leonard HL, Lenane MC, Swedo SE, Rettew DC, Gershon ES, Rapopport JL. Tics and Tourette's Disorder: a 2- to 7- year Follow-up of 54 Obsessive-Compulsive Children. Am J Psychiatry 1992;149:1244-51.

11. Lenane MC, Swedo SE, Leonard H, Pauls DL, Sceery W, Rapoport JL. Psychiatric disorders in first degree relatives of children and adolescents with obsessive-compulsive disorder. J Am Acad Child Adolesc Psychiatry 1990;29:407-12.

12. Riddle MA, Scahill L, King R. Obsessive-compulsive disorder in children and adolescents: phenomenology and family history. J Am Acad Child Adolesc Psychiatry 1990;29:766-72.

13. Leonard HL, Lenane MC, Swedo SE, Rettew DC, Gershon ES, Rapopport JL. Tics and Tourette's Disorder: a 2- to 7- year follow-up of 54 Obsessive-Compulsive Children and adolescents. Arch Gen Psychiatry 1993;50:429-39.

14. Pauls DL, Alsobrook JP, Goodman W, Rasmussen S, Leckman JF. A family study of obsessive-compulsive disorder. Am J Psychiatry 1995;152:76-84.

15. Pauls DL, Raymond CL, Stevenson JM, Leckman JF. A family study of Gilles de la Tourette syndrome. Am J Hum Genet 1991;48:154-63.

16. Rosario-Campos MC, Mercadante MT. Transtorno obsessivocompulsivo. Rev Bras Psiquiatria 2000;22(Supl 2):16-9.

17. Grados M, Scahill L, Riddle MA. Pharmacotherapy in children and adolescents with obsessive-compulsive disorder. Child Adolesc Psychiatr Clin N Am 1999;8:617-34.

18. Ackerman DL, Greenland S, Bystritsky A, Morgenstern H, Katz RJ. Predictors of treatment response in Obsessive-Compulsive disorder: multivariate analyses from a multicenter trial of clomipramine. J Clin Psychopharmacol 1994;14:247-54.

19. Ravizza L, Barzega G, Bellino S, Bogetto F, Maina G. Predictors of drug treatment response in obsessive-compulsive disorder. J Clin Psychiatry 1995;56:368-73.

Correspondência: Maria Conceição do Rosario-Campos

Yale Child Study Center - 230 South Frontage Road, Room \# I-267 - 06520 New Haven, CT, USA Tel.: (00xx1) (203) 785-6070-Fax:(00xx1) (203) 785-7611 -Email: maria.rosario-campos@yale.edu 